\title{
Modulation of trained and extinguished stimuli by facilitators and inhibitors
}

\author{
DALE SWARTZENTRUBER and ROBERT A. RESCORLA \\ University of Pennsylvania, Philadelphia, Pennsylvania
}

\begin{abstract}
Pigeon subjects received training of a diffuse stimulus as either a conditioned inhibitor or a conditioned facilitator of a keylight signal for food. The ability of these diffuse stimuli to modulate responding was then assessed with two other keylights that were undergoing discrimination reversal. At a point where responding was equivalent for the two targets, the modulators had a greater impact on the target undergoing extinction than on the target undergoing acquisition. These results have implications for the nature of modulation and extinction.
\end{abstract}

Considerable recent interest has focused on the ability of one Pavlovian stimulus to modulate responding to another. Several Pavlovian procedures appear to generate stimuli that do not simply signal the unconditioned stimulus (US), but instead set the occasion for the relationship between another stimulus and the US. Perhaps the two most frequently studied instances are conditioned inhibition (feature negative) and conditioned facilitation (feature positive). In a conditioned inhibition procedure, one stimulus (A) is followed by the US except when it is accompanied by another stimulus (X). The result of such a procedure is that $A$ evokes a response but $X$ inhibits that response to $\mathrm{A}$. In a conditioned facilitation procedure, the converse contingencies are arranged so that $\mathrm{A}$ is followed by the US only when it is accompanied by X. Under some circumstances, such a procedure results in responding to A only in the presence of $\mathrm{X}$.

A major focus for studies of such modulation has been the degree to which $\mathrm{X}$ will transfer its modulation from $\mathrm{A}$, the original training target, to another target stimulus, B. Successful transfer has been taken as indicating that $\mathrm{X}$ has some general property, perhaps extending to various B stimuli paired with the same US as that with which A has been paired (e.g., Rescorla, 1985). Failure of transfer has been taken as indicating that $X$ acts more specifically on A, perhaps by affecting A's associations (e.g., Holland, 1983).

Because of the diagnostic potential of transfer, there have been a number of investigations of the nature of the target stimuli to which X might transfer. Studies of modulatory transfer have been conducted on both facilitative and inhibitory learning; however, most of this work has concentrated on the ability of a facilitatory $\mathrm{X}$ to improve

This research was supported by National Science Foundation Grant BNS-88-03514 to R.A.R. and National Institute of Mental Health postdoctoral training Grand MH 09896 to D.S. Correspondence concerning this article should be addressed to D. Swartzentruber, who is at the Department of Psychology, Ohio Wesleyan University, Delaware, OH 43015 (e-mail: deswartz@cc.owu.edu). responding to a transfer target, $\mathrm{B}$. Although in some situations, $\mathrm{X}$ appears to be highly constrained to modulate only the A stimulus with which it has been trained (e.g., Holland, 1983, 1986), in other instances X acts on stimuli with various other training histories. For instance, a $B$ stimulus that has been the target of another modulator, $\mathrm{Y}$, will frequently act as an especially receptive target for X (e.g., Holland, 1989a, 1989b). Similarly, in some preparations, a B stimulus that has simply undergone excitatory conditioning and then been subjected to extinction is a favorable target for a facilitatory X (e.g., Rescorla, 1985, but see, e.g., Holland, 1986). By contrast, stimuli that have received simple Pavlovian excitatory training or that have never been trained show little evidence of being good targets for facilitatory modulation. It is difficult to summarize the mixed results from positive and negative modulatory transfer experiments. There is general agreement, however, that both positive and negative modulators will transfer control across targets, and the extent of transfer depends heavily on the conditioning history of the transfer target.

The goal of the present experiments was to provide a more systematic comparison for two types of targets: a stimulus that has been trained and partially extinguished and a stimulus that has received simple excitatory training after nonreinforced preexposure. Holland (1991) and Holland and Reeve (1991), using a discrete-trial instrumental situation, both found excellent transfer to a trained and extinguished stimulus, and Rescorla (1985), using an autoshaping procedure, found superior transfer to an extinguished stimulus compared with one that had simply received training. On the other hand, Holland (1989a), using an appetitive Pavlovian procedure with an observational dependent variable, failed to find transfer to trained and extinguished stimuli but Davidson and Jarrard (1989) succeeded with a similar procedure.

In addition to yielding varying results, these experiments each had procedural shortcomings. For instance, none of the experiments satisfactorily matched the current level of responding to a B target that had received 
simple training and a B target that had been trained and extinguished. As a result, apparent differences in sensitivity of various target types may simply reflect differences in the levels of responding they control when presented alone. Similarly, several of these studies failed to incorporate control stimuli that were treated like the facilitators but that had not been informative with regard to the treatment of their targets. Consequently, they failed to demonstrate that differences in modulation of responding depended on the facilitatory training of X. Moreover, these studies failed to allow comparison of the two target types in the same animal at the same time, thereby leaving open the possibility that any differences observed lie not in the specific training of $B$ but in the general treatment of the animal.

The present experiments were designed to correct these shortcomings and so to provide a more precise comparison of the responsiveness to modulation of a stimulus that is acquiring excitation and a stimulus that is undergoing extinction. The strategy adopted was like that employed by Holland and Reeve (1991) for stimuli given ambiguous cue training. A trained modulator was repeatedly transferred to two stimuli, $\mathrm{C}$ and $\mathrm{D}$, during $\mathrm{C}-/ \mathrm{D}+$ training. Because the animal had previously received $C+/ D-$ training, this meant that transfer was assessed while $C$ was undergoing extinction and $D$ was undergoing original excitatory conditioning. At some point during this reversal training, the levels of responding to $\mathrm{C}$ and $\mathrm{D}$ were expected to be quite similar, despite their different training histories. The question of interest was the degree to which the modulators affected performance to these targets when responding to the targets alone was at this common level. Importantly, transfer by the modulators was compared to the effect of control stimuli that had been treated like the modulators but that had been uninformative with regard to the treatment of their target stimuli. In order to ensure close matching of the levels of responding to $C$ and $D$, the point of their similar response rates was determined for each animal and transfer was assessed at that point during reversal training.

Experiment 1 carried out this transfer assessment for a facilitative modulator. As noted above, there is some empirical reason to expect a difference in transfer when a facilitator is used. Moreover, there are theoretical grounds for anticipating that result; Rescorla (1985) has argued that facilitators function by removing inhibition, such as that which might develop during extinction. Hence one would expect that a facilitator would promote responding to an extinguishing stimulus (by removing its inhibition) but fail to act on a stimulus undergoing initial training (which presumably lacks an inhibitory component). Experiment 2 carried out a similar procedure with an inhibitory modulator. Although there are several studies examining the effect of facilitators on various targets, there are fewer instances in which the effects of inhibitors have been examined for various tar- gets. Moreover, current theories of inhibition provide little clear guidance. Hence it is of special importance to examine whether inhibitors transfer differentially to different targets.

Both experiments used autoshaping procedures in which pigeon subjects received food following the brief illumination of a keylight and in which conditioning was assessed by the rate of pecking at that keylight. In each case the modulatory effect of a diffuse auditory or visual $\mathrm{X}$ was assessed by its ability to enhance or depress responding to keylight target stimuli.

\section{EXPERIMENT 1}

In this experiment, a diffuse auditory or visual stimulus, $\mathrm{X}$, was first trained as a facilitator. A 5-sec keylight, A, was presented either alone (without food), or at the end of the 15-sec diffuse $X$ stimulus (and accompanied by food). After this $\mathrm{A}-/ \mathrm{XA}+$ training, $\mathrm{X}$ was tested for its transfer to two other targets, one undergoing original acquisition and one undergoing extinction.

Rescorla (1985) conducted a preliminary experiment of this sort and found that the facilitator successfully transferred only to the stimulus undergoing extinction. However, that experiment assessed transfer to the same target during its initial acquisition and then during its subsequent extinction. As a result it confounded the associative history of the target with its overall familiarity. Moreover, Rescorla failed to include any control stimulus against which to evaluate the effect of the facilitator and hence was unable to identify the modulatory effect of the facilitator as due to its own training history.

In the present experiment, a facilitator was transferred to two different targets that were equated both in familiarity and in level of responding but that differed in their conditioning history. This was accomplished by first carrying out simple discrimination training with the targets, $\mathrm{C}+\mathrm{D}-$, and then testing the facilitator during the course of reversal training, $\mathrm{C}-/ \mathrm{D}+$. The data of interest come from the point in the reversal at which the response rates to $\mathrm{C}$ and $\mathrm{D}$ are equivalent. The issue is the degree to which the facilitator can promote responding to those stimuli from that common response baseline.

So that the importance of the particular training history of the facilitator could be evaluated, all animals also received training with a second diffuse stimulus, $Y$, in a "pseudofacilitator" design. In this procedure, a 5-sec keylight target, B, was reinforced both when presented alone and when presented at the end of the 15 -sec Y. In such a $\mathrm{B}+/ \mathrm{YB}+$ procedure, $\mathrm{Y}$ is uninformative about the treatment of $B$ but it has a keylight reinforced in its presence in the same manner as does the $\mathrm{X}$ facilitator. It is common to compare the impact of a facilitator with that of a pseudofacilitator control to assess the contribution of the training procedure used to make $\mathrm{X}$ facilitatory (see, e.g., Rescorla, 1985).

The design of Experiment 1 is summarized in Figure 1. 


\begin{tabular}{l|c|c} 
Fac Train & Discrim & Reversal \& Test \\
\hline$X A+, A-$ & $C+$ & $C-, X C, Y C$ \\
$Y B+, B+$ & $D-$ & $D+, X D, Y D$
\end{tabular}

Figure 1. Design of Experiment 1. A diffuse $X$ was trained as a facilitator with the target keylight $A$, whereas $Y$ was trained as a control stimulus with another target $B$. During reversal training on $\mathbf{C}$ and $D$ keylight targets, responding was tested to $C$ and $D$ in combination with $X$ and $Y$.

\section{Method}

Subjects. The subjects were 16 experimentally naive female Carneaux pigeons, about 1 year old. The subjects were housed in pairs and maintained at $80 \%$ of their free-feeding weights.

Apparatus. Eight identical operant chambers, each measuring $27 \times 27 \times 35 \mathrm{~cm}$, were used. One wall of each chamber was constructed of aluminum and contained a $5 \times 5 \mathrm{~cm}$ food magazine centered $5 \mathrm{~cm}$ above the wire mesh floor. A $11.8 \times 14.5 \mathrm{~cm}$ response key, constructed of clear Lucite acrylic, was centered $9 \mathrm{~cm}$ above the magazine, behind a $10 \times 8 \mathrm{~cm}$ rectangular opening in the chamber wall. A Magnavox (Model CK3923) color television was located $0.2 \mathrm{~cm}$ behind the response key. A computer was programmed to generate and display four 5-sec visual stimuli on the television screen: a 0.9 -cm-diameter red circle, a $0.8 \times 0.8 \mathrm{~cm}$ blue square, a $1.4 \times 0.2 \mathrm{~cm}$ yellow horizontal line, and a green triangle with $1.0-\mathrm{cm}$ sides and a $0.8-\mathrm{cm}$ base.

The other three walls and ceiling of the chambers were constructed of clear Plexiglas. The chambers were placed in soundand light-attenuating shells with ventilating fans providing background noise of $62 \mathrm{~dB}(\mathrm{~A})$. A $6-\mathrm{W}$ bulb was located on the wall of the shells, above the chamber ceiling. This houselight was continuously illuminated except during operation of the food hopper, during which a 6-W bulb illuminated the inside of the hopper and Purina Pigeon Grain was made available. The houselight could be interrupted at the rate of $2 / \mathrm{sec}$ to provide a 15 -sec diffuse visual signal. Speakers were also mounted on the wall of the shells, allowing the presentation of a $15-\mathrm{sec}$ white noise, which raised the sound level to about $80 \mathrm{~dB}(\mathrm{~A})$.

Procedure. The subjects were initially given magazine training. On the 1st day, they were placed in the chambers with the hoppers raised. After they had eaten for approximately $15 \mathrm{sec}$, the hoppers were briefly lowered. Then the hoppers were made available for gradually shorter times, spaced at longer intervals, until the birds promptly ate during a $5-\mathrm{sec}$ presentation. On each of the next 2 days, the birds received forty-four 5-sec hopper presentations. During these sessions and all subsequent phases of the experiment, the intertrial interval (ITI) was variable; with a mean of $1 \mathrm{~min}$.

On each of the next 18 days, the birds received initial autoshaping training. In each session, they received 32 reinforced presentations of a $1.8-\mathrm{cm}$ white circle. For the next two sessions, the birds received a nonreinforced pretest of all six stimuli to be used in the experiment. These sessions contained 8 presentations of each stimulus.

For the next 12 sessions, the birds received training in which one of the diffuse stimuli (X) served as a facilitator for a keylight target (A). Each session contained 12 nonreinforced presentations of $\mathrm{A}(\mathrm{A}-)$ and 12 reinforced presentations of $\mathrm{A}$ given during the last $5 \mathrm{sec}$ of the $15-\sec \mathrm{X}(\mathrm{XA}+)$. The other diffuse stimulus $(\mathrm{Y})$ was trained as a "pseudofacilitator" of $B$. This treatment consisted of 12 reinforced presentations each of $\mathrm{B}$ alone and during the last 5 -sec of the 15-sec Y. As a result of these treatments, X signaled trials on which $A$ was reinforced, whereas $Y$ had an equivalent number of presentations but provided no information about reinforcement of $B$. For half of the animals, $\mathrm{X}$ was the noise and $\mathrm{Y}$ was the light, respectively; for the other half, the roles of these stimuli were interchanged. For half of each of these subgroups, A was the red circle and $\mathrm{B}$ was the blue square; for the other half, these roles were interchanged. On reinforced trials, food presentation consisted of 5-sec availability of grain.

The next two sessions each consisted of 16 additional pretest presentations of the $C$ and $D$ stimuli, the yellow horizontal line and the green triangle. Their roles as $\mathrm{C}$ and $\mathrm{D}$ were completely counterbalanced across the four previous subgroups.

The next six sessions consisted of discrimination training with $\mathrm{C}$ and $\mathrm{D}$. In each session, there were $16 \mathrm{C}+$ presentations and 16 $\mathrm{D}-$ presentations. In each of the following three sessions, facilitation and pseudofacilitation training were continued as in previous sessions. Beginning in the next session, the ability of $\mathrm{X}$ and $\mathrm{Y}$ to transfer control of responding to $C$ and $D$ was examined. The first session began with six randomly intermixed presentations of $\mathrm{XA}+, \mathrm{A}-, \mathrm{YB}+$, and $\mathrm{B}+$, and four presentations each of $\mathrm{C}+$ and $\mathrm{D}-$. Test trials then consisted of two presentations each of $\mathrm{XC}-$, $\mathrm{YC}-, \mathrm{C}+, \mathrm{XD}-, \mathrm{YD}-$, and $\mathrm{D}-$.

For the next five sessions, transfer of $X$ and $Y$ to $C$ and $D$ was assessed while the reinforcement contingencies for $C$ and $D$ were reversed. Each session contained two blocks of trials consisting of two each of the original facilitation and pseudofacilitation training trials, four each of $\mathrm{C}-$ and $\mathrm{D}+$, and one test trial each of $\mathrm{C}$ and $\mathrm{D}$ presented during $\mathrm{X}$ and $\mathrm{Y}$. The various trial types were intermixed, and the order of the four test trials was counterbalanced across subjects. The trial blocks on which responding to $\mathrm{C}$ and $\mathrm{D}$ crossed were determined for each animal. The effects of the diffuse stimuli during those trials blocks were the primary data of interest.

\section{Results}

Facilitation training proceeded smoothly. On the final day of training, the mean rate of keypecking to $A$ on $\mathrm{XA}+$ trials was 148.6 responses per minute, whereas when $\mathrm{A}$ was presented alone the mean rate was only 36.4. Thus, $\mathrm{X}$ reliably facilitated responding to A [Wilcoxon $T(9)=0$ ]. As expected, the mean rates to $B$ were comparable whether $B$ was presented during the pseudofacilitator $Y, 140.9$, or alone, $138.4[T(13)=30]$. There were no reliable differences between the light and the noise in their roles as facilitator or pseudofacilitator [Mann-Whitney $U \mathrm{~s}(7,7)>27]$.

On the final session of $\mathrm{C}+\mathrm{D}-$ training, the mean rates of responding to $C$ and $D$ were 192.5 and 1.4 responses per minute $[T(14)=0]$. This session contained an initial assessment of modulatory transfer to $C$ and $D$. The mean rates of responding to $\mathrm{C}$ during the facilitator, $\mathrm{X}$, and the pseudofacilitatory control, $\mathrm{Y}$, were 183.2 and 183.2 responses per minute, respectively. Responding to $\mathrm{C}$ was not affected by either $\mathrm{X}[T(12)=25]$, or $\mathrm{Y}[T(11)$ $=24.5]$. Response rates to $D$ during $X$ and $Y$ were 29.1 and 1.8 , respectively. Responding during $X$ was observed in only 5 of the 14 subjects and was not reliably greater than that to $\mathrm{D}$ alone $[T(5)=2]$. Responding during $\mathrm{Y}$ was observed in only 1 subject.

During the next five sessions, in which $\mathrm{C}$ received extinction and $D$ received initial excitatory acquisition, responding to $\mathrm{C}$ declined and that to $\mathrm{D}$ increased. However, the rates at which these changes took place varied widely across subjects. Consequently, the rates of responding for individual subjects were examined during each half-session block to determine the point at which 
$\mathrm{C}$ and $\mathrm{D}$ elicited equivalent rates of responding. The test trials with $X$ and $Y$ that were conducted during these blocks were then used in the assessment of modulatory transfer. For each subject, the last block during which C elicited more responding than $\mathrm{D}$ and the first block in which $\mathrm{D}$ elicited more responding than $\mathrm{C}$ were both identified. The response rates from these trial blocks and, where necessary, all trial blocks intervening between these two were then used in the analysis. Across subjects, these calculations included trials ranging from the second half of the first session to the second half of the fifth session. The mean number of trial blocks used was 4.2 ; the mean point of crossing occurred between the fifth and sixth trial blocks. Two subjects failed altogether to respond to $D$; data from these subjects were excluded from the analyses.

Figure 2 shows the data from the blocks during which $\mathrm{C}$ and $\mathrm{D}$ crossed. The figure shows the mean rates of responding to $C$ and $D$ alone, and to $C$ and $D$ when presented during the facilitator, $X$, and the pseudofacilitator, $Y$. As is suggested by the figure, the analysis was successful in identifying blocks on which $C$ and $D$ were comparable in response rate $[T(11)=30]$. However, the effects of the facilitator and pseudofacilitator were quite different for these two stimuli despite their similar baseline. The facilitator reliably enhanced responding to the extinguished target, $\mathrm{C}$, compared with the target alone $[T(12)=8]$, whereas a similar enhancement was not observed on the excitatory stimulus, D $[T(11)=34]$. Furthermore, the effect of the facilitator on $\mathrm{C}$ was reliably greater than that on $\mathrm{D}[T(13)=19]$. On the other hand, the pseudofacilitator had no reliable effect on either $C[T(13)=26]$ or $D[T(13)=23]$, and there was no difference in the magnitudes of these effects $[T(11)=30]$.

An important feature of the present experimental design is that it allowed a direct comparison of the sensitivities of extinguishing and excitatory targets to modu-

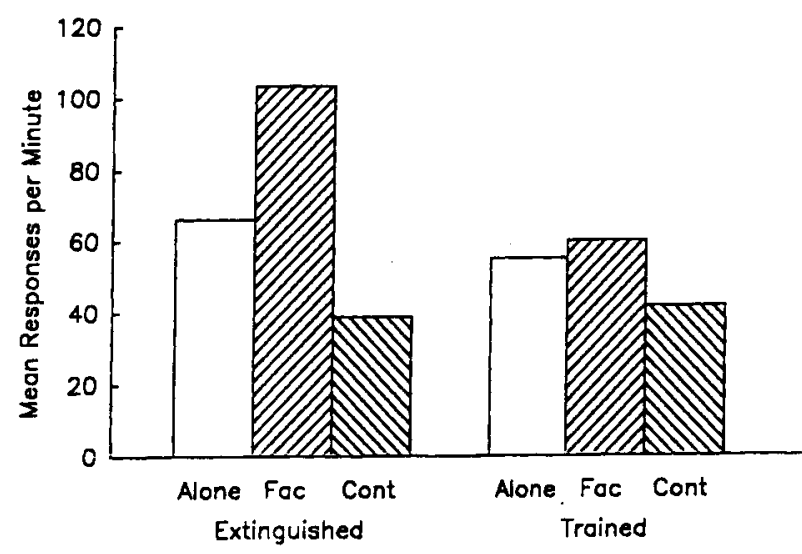

Figure 2. Results of the transfer test in Experiment 1. Responding is shown separately for an extinguished (C) stimulus and a trained stimulus (D) when they were presented alone, in combination with a facilitator $(X)$, and in combination with a control stimulus (Y). latory transfer in a situation where responding was assessed during both a modulator and a control stimulus. Thus, in order to compare these two targets directly, a measure of each target's sensitivity was calculated as the difference in response rate between these two test compounds. The facilitator produced reliably greater responding to the extinguished $\mathrm{C}$ stimulus than did the pseudofacilitator $[T(13)=10.5]$, and there was no reliable difference between the facilitator and pseudofacilitator in their effects on the excitatory D stimulus $[T(12)=15]$. Most importantly, the difference in responding during $\mathrm{X}$ and $\mathrm{Y}$ for the extinguished stimulus was reliably greater than it was for the excitatory stimulus $[T(13)=11.5]$, suggesting that an extinguishing stimulus, $\mathrm{C}$, is more sensitive to facilitatory control than is a stimulus undergoing initial excitatory conditioning, D.

\section{Discussion}

The results of this experiment generally confirm the findings reported by Rescorla (1985). A facilitator transferred successfully to a stimulus undergoing extinction but not to one undergoing initial excitation. This occurred at a time when the levels of responding to those stimuli were matched on an individual animal basis. Hence the difference depends on the training history of the target stimuli, not the current level of performance they control. Moreover, the fact that this transfer was evaluated in relation to that obtained from a control pseudofacilitator suggests that the special sensitivity of a stimulus undergoing extinction depends on the training history of the modulator. It is not simply that extinguishing stimuli are more sensitive to the effects of any superimposed stimulus. These observations greatly strengthen the claim that trained and extinguished stimuli are especially sensitive targets for modulation by facilitators.

One account of this special sensitivity of extinguished stimuli can be given in terms of facilitators acting by the removal of inhibition. For instance, Rescorla (1985) followed Konorski (1948) in suggesting that one effect of extinction is to give a stimulus the ability to raise the threshold for activation of the representation of the US. According to this view, an extinguished stimulus retains its excitatory association with the US but also takes on the ability to raise the threshold for activation of the US. Facilitators might act by lowering that artificially raised threshold, hence revealing the underlying excitatory association and producing responding. A target stimulus that had received only initial training would not raise the threshold for US activation and hence would be less susceptible to an agent that lowered that threshold.

\section{EXPERIMENT 2}

This experiment assessed transfer of inhibition that was parallel to that conducted for facilitation in Experiment 1. Although some investigators (e.g., Rescorla, 1993) have used partially extinguished stimuli as targets 
for assessing inhibition, there are only a few direct comparisons of the sensitivity of different target types to modulation by an inhibitor (e.g., Holland \& Lamarre, 1984; Lamarre \& Holland, 1987). Experiment 2 provided such a comparison for stimuli undergoing extinction and acquisition. Initially, two diffuse stimuli were trained, one as a conditioned inhibitor using an $\mathrm{A}+/ \mathrm{XA}-$ procedure, and the other as a pseudoinhibitor using a $\mathrm{B}-/ \mathrm{YB}-$ procedure. Then these stimuli were transferred to $C$ and $D$, which were undergoing reversal training. As in Experiment 1, the question of interest is the degree to which $\mathrm{X}$ and $\mathrm{Y}$ would affect responding to $\mathrm{C}$ and $\mathrm{D}$ at a time when the response level is the same for $\mathrm{C}$ and $\mathrm{D}$.

\section{Method}

Subjects and Apparatus. The subjects were 15 experimentally naive female Carneaux pigeons, about 1 year old. They were maintained and housed in the same manner as were the subjects in Experiment 1. The apparatus was the same as in Experiment 1.

Procedure. The subjects received the same magazine and autoshaping training as in Experiment 1. On the next session, the birds received eight pretest presentations of each of the six experimental stimuli.

For the next four sessions, the birds received discrimination training with the two stimuli, $A$ and $B$, that were to be used as the targets for initial inhibition and pseudoinhibition training, respectively. These sessions contained 16 reinforced presentations of $A$, and 16 nonreinforced presentations of B. As in Experiment 1, A and $B$ were the red circle and the blue square, counterbalanced.

Inhibition and pseudoinhibition training were conducted over the next 20 sessions. The training parameters were analogous to those in Experiment 1, with 12 presentations each of $\mathrm{XA}-, \mathrm{A}+$, $\mathrm{YB}-$, and $\mathrm{B}-$. As a result, $\mathrm{X}$ was established as an inhibitor of responding to $A$, and $Y$ received similar exposure but provided no information about the treatment of $B$. The identities of the diffuse $\mathrm{X}$ and $\mathrm{Y}$ stimuli were counterbalanced across animals.

In the following session, there were 16 additional nonreinforced pretest presentations of the $C$ and $D$ transfer targets. These stimuli consisted of the yellow horizontal line and the green triangle, counterbalanced with regard to all prior stimulus assignments. Then $C$ and $D$ underwent discrimination training. There were 16 $\mathrm{C}+$ and $16 \mathrm{D}-$ presentations in each of eight sessions. One further session of inhibition and pseudoinhibition training followed.

In each of the next five sessions, the sensitivity of $C$ and $D$ to modulatory control by $\mathrm{X}$ and $\mathrm{Y}$ was examined by using procedures analogous to those of Experiment 1 . The first session began with an intermixing of four trials each of $\mathrm{C}+$ and $\mathrm{D}-$ with six each of the inhibition and pseudoinhibition training trials. Test trials then consisted of two presentations each of $\mathrm{XC}-, \mathrm{YC}-, \mathrm{C}+, \mathrm{XD}-$, YD-, and $\mathrm{D}-$. In the next four sessions, test trials were conducted concurrently with $\mathrm{C}-/ \mathrm{D}+$ reversal training. The testing parameters were analogous to those of Experiment 1 .

\section{Results}

Over the course of inhibition training, responding to the A target alone remained high, whereas responding to A decreased in the presence of X. Response rates on A+ and XA - trials were 169.5 and 22.5 , respectively, on the final day of training $[T(11)=0]$. Responding to $\mathrm{B}$ remained low both when $B$ was presented alone and when it was in the presence of Y. On the final day of training, the mean rates of responding were 5.8 and 8.2 , respectively. There were no reliable differences between the light and the noise in their roles as inhibitor or pseudoinhibitor [Mann-Whitney $U s(6,6)>6$ ].

In the final session of $\mathrm{C}+/ \mathrm{D}-$ training, the mean rates of responding to $C$ and $D$ were 198.5 and $0.0[T(12)=$ $0]$. In the initial test of modulatory transfer, the mean rates of responding to $\mathrm{C}$ during the inhibitor, $\mathrm{X}$, and the pseudoinhibitor, Y, were 160.4 and 170.2 , respectively. Although both stimuli suppressed responding to $\mathrm{C} \mathrm{nu}-$ merically, responding was not reliably suppressed by either X $[T(10)=18]$ or $\mathrm{Y}[T(11)=21]$. During the analogous tests with $\mathrm{D}$, responding was observed in only 1 subject.

During the next four sessions, in which $\mathrm{C}$ was extinguished and $\mathrm{D}$ acquired excitation, responding by individual subjects was examined by using the same technique as in Experiment 1. Three subjects failed to respond more to $\mathrm{D}$ than to $\mathrm{C}$; data from these subjects were excluded from all analyses.

Figure 3 shows the data from the point at which $\mathrm{C}$ and $\mathrm{D}$ crossed. The figure shows the mean rates of responding to $C$ and $D$ alone, and to $C$ and $D$ when they were presented during the inhibitor, $X$, and the pseudoinhibitor, $Y$. As expected, the rates of responding to $\mathrm{C}$ and $\mathrm{D}$ were comparable $[T(10)=21]$. However, the inhibitor and control pseudoinhibitor generated different patterns of responding to $C$ and $D$. When compared with responding to the targets presented alone, the inhibitor suppressed responding to $C[T \mathrm{~s}(11)=0]$ but not to $\mathrm{D}$ $[T(11)=11]$. Furthermore, this suppression was reliably greater to $\mathrm{C}$ than to $\mathrm{D}[T(11)=3]$. The pseudoinhibitor also suppressed responding to $C[T(11)=9]$ and not $D$ $[T(10)=16]$, but the difference in the magnitudes of these effects was not reliable $[T(11)=16]$.

In order to compare the sensitivities of the extinguished and excitatory targets directly, the differences in responding during the inhibitor and pseudoinhibitor were calculated for each target. The inhibitor produced reliably greater suppression of the extinguished target than did the pseudoinhibitory control $[T(11)=0]$, whereas responding to the excitatory target was comparable in the

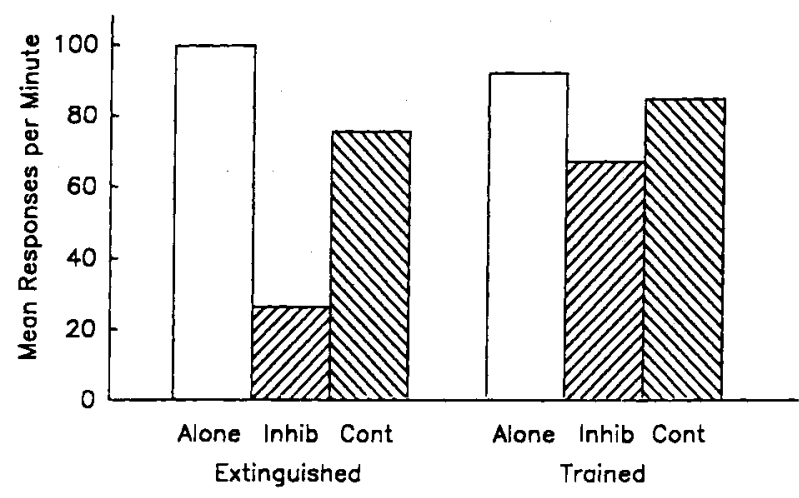

Figure 3. Results of the transfer test in Experiment 2. Responding is shown separately for an extinguished stimulus (C) and a trained stimulus (D) when they were presented alone, in combination with an inhibitor (X), and in combination with a control stimulus (Y). 
presence of either stimulus $[T(9)=11]$. Of most importance, as in Experiment 1, the difference in the of the inhibitor and pseudoinhibitor on the extinguished target was reliably greater than the analogous difference in the effects on the excitatory target $[T(11)=9]$, suggesting that an extinguishing stimulus is more sensitive to inhibitory modulation than is an excitatory stimulus.

\section{Discussion}

The results of this experiment are parallel to those of Experiment 1 . A diffuse inhibitor successfully transferred to a target stimulus that was undergoing extinction but showed less transfer to a target undergoing excitatory training. Moreover, this greater transfer was evaluated relative to a control stimulus with a similar history but lacking in inhibitory training. Consequently, these data suggest that the sensitivity of an extinguishing target to modulation is special both to the training history of the target and to the inhibitory training history of the modulator. Although previous results have indicated that facilitators transfer more successfully to extinguishing stimuli, the finding that inhibitors also transfer better to such targets has not previously been reported.

It may seem somewhat surprising that the present experiment found no reliable transfer of inhibition to an excitatory target that had not undergone extinction. Such a transfer procedure is routinely used to detect inhibition in a variety of preparations. However, several points should be noted. First, the inhibitor did produce a numerical depression in responding relative to both the target alone and the control stimulus, although this depression was not reliable. Second, the present excitor had undergone a phase of nonreinforced exposure prior to excitatory conditioning. We know of no research examining the effect of preexposure on sensitivity to inhibitory control. Third, most transfer tests of inhibition are conducted under conditions of extinction. Over the course of the transfer test, the target excitor typically receives nonreinforced presentations and hence is subjected to extinction. It seems likely that this treatment allows the target to develop enhanced sensitivity in many tests of the transfer of inhibition.

\section{GENERAL DISCUSSION}

The results of these experiments suggest that extinguished stimuli are especially sensitive transfer targets for modulation both by facilitators and inhibitors. These results have implications for theories of modulation, for the detection of modulation, and for the effects of extinction.

\section{Theories of Modulation}

Perhaps the simplest account of modulation is given in terms of summation of the excitatory and inhibitory strengths of the individual elements (e.g., Bombace, Brandon, \& Wagner, 1991; Rescorla \& Wagner, 1972). According to such a view, responding to an $A B$ compound can be understood in terms of the summation of the strengths of the A and B elements. As both Holland $(1983,1985)$ and Rescorla $(1985,1991)$ note, there are many reasons to believe that this cannot be a complete account of modulation. For instance, manipulations that change the excitatory strength of a modulator frequently have no effect on its ability to modulate responding. Perhaps most striking is the observation (Rescorla, 1991) that excitatory training can even enhance the modulatory power of a conditioned inhibitor. The results of the current experiments present many summation models with a related problem. The similarity of responding to $\mathrm{C}$ and $\mathrm{D}$ at the point of testing would be interpreted by most models in terms of their having similar net associative strengths. Yet the extinguished stimulus and the simple excitor entered into putative summation in quite different ways. Even models that assume that extinction leaves original excitation intact seem poorly prepared for this difference in summation from stimuli with similar net values.

A comparable difficulty confronts attempts to account for transfer of modulation in terms of generalization to the test $\mathrm{XC}$ and $\mathrm{XD}$ compounds from the training XA compound. If $C$ and $D$ are matched on their net associative strengths and are balanced in their perceptual similarity to A, it is not clear how they could receive different amounts of generalization from the XA compound. For instance, one current approach (Pearce, 1987) attempts to account for modulation in terms of such generalization. But simulations of that theory found that it failed to anticipate the difference in susceptibility of $\mathrm{C}$ and $\mathrm{D}$ to modulation. One approach to elaborating on this theory might be to view a common training history as enhancing the similarity between the training target and certain transfer targets (cf. Honey \& Hall, 1989). One might argue, for instance, that the mixture of reinforcement and nonreinforcement that $A$ receives during $\mathrm{A}+/ \mathrm{XA}-$ inhibition treatment makes it more similar to a target stimulus that has itself received first reinforcement and then nonreinforcement. The details of such treatment-mediated similarity need to be worked out; however, even with such an elaboration, the model suggested by Pearce makes no fundamental distinction between modulation and excitation. Hence, it cannot account for the failure of excitatory manipulations of the modulator to affect its modulatory power.

As noted previously, the threshold-shifting account proposed by Rescorla (1985) provides a natural description of the special sensitivity of an extinguished stimulus to facilitation. The facilitator acts to reduce an artificially elevated threshold for activation of the US. But it is not at all clear that this approach can provide an equally natural account of the similar sensitivity of an extinguished stimulus to the action of an inhibitor. In fact, one might anticipate that a stimulus with an artificially elevated threshold would be less sensitive to attempts of other stimuli to further elevate that threshold.

The hierarchical view of modulation suggested in various forms by Holland $(1983,1989$ b) also has difficulties with these data. As Holland and Reeve (1991) have 
noted, some versions of that approach anticipate no transfer at all of modulation to new targets, aside from that based on stimulus generalization. But the present data imply that generalization cannot provide a complete account of transfer.

One might provide a general account of the present results within a retrieval view of modulation (e.g., Bouton, 1991). According to that view, the job of the modulator is to selectively retrieve one of various associative relations that have been acquired by the target. Hence, after $\mathrm{A}-/ \mathrm{XA}+$ training, the $\mathrm{X}$ stimulus activates the $\mathrm{A}-\mathrm{US}$ association rather than the $\mathrm{A}-$ association and so promotes responding. On this account, stimuli that have multiple associations, such as an extinguished stimulus, would be especially susceptible to modulation because the modulator would have multiple associations from which to choose. Notice, however, that both $\mathrm{C}$ and $\mathrm{D}$ had received both reinforced and nonreinforced experiences in the present experiments; they differed only in the order of those treatments. Hence, in order to account fully for the present data, a retrieval view would have to assume that the effects of nonreinforcement are different when it occurs before, versus after, reinforcement. However attractive this general framework may be, the rules for retrieval and the mechanisms by which a stimulus gains the ability to produce retrieval are yet to be worked out. Until that is done, a retrieval account remains at a different level of discourse than that of other available theories of modulation.

\section{Implications for Detecting Modulation}

The observation that extinguished stimuli are especially sensitive to modulation has practical implications for assessing modulation. It suggests that not all stimuli are equally effective targets for studies that are designed to examine the presence of modulation.

One elementary implication is that one cannot safely use the degree to which a modulator affects its own training target as a good index of its degree of modulation. Procedures intended to vary the nature of the modulatory training may as well vary the treatment of the target and hence the ability to measure modulation. For instance, comparison of the inhibitory power of $X$ after an $\mathrm{A}+/ \mathrm{XA}$ - training procedure with that of an $\mathrm{X}$ after an $\mathrm{A}+\mathrm{X}$ - procedure cannot be made by looking at responding to the XA compound; those treatments differentially treat A and so may make it differentially susceptible to modulation by X. Even if the two procedures established equivalent inhibition to $\mathrm{X}$, they might produce different degrees of modulation to the differentially treated As. Similarly, procedures that vary the temporal relation between $\mathrm{X}$ and $\mathrm{A}$ in an $\mathrm{A}-/ \mathrm{XA}+$ procedure may well result in different treatments of $A$ that confound estimates of X's modulatory power that can be obtained by looking at responding to the XA compound. Consequently, the present results make it especially important that modulation be assessed to a common transfer target with a common history of treatment (cf. Rescorla, 1989).
Perhaps less obviously, these results also suggest that one must be cautious in concluding that a treatment has or has not established modulation from data based on transfer to a particular target type. Although discussions of inhibition have not emphasized the importance of target treatment, summation tests carried out with different targets may yield quite different results. For instance, in experiments intended to assess the inhibitory power of a context, inspecting the impact on a simple excitor may be inadequate. Possibly a better target would be a trained and extinguished stimulus. Indeed, Bouton and Swartzentruber (1989) reported results suggesting that what might be interpreted as inhibitory control by a context can be detected better by its effects on a trained and extinguished stimulus than by its effects on a simple excitor.

The present results also reemphasize the importance of control stimuli in assessing the impact of a modulator. In both of the present experiments, the control stimulus had a small depressive effect on responding to the target. That effect presumably reflects the unconditioned impact of the control stimulus that is to be expected in the absence of modulatory treatment. That is, there are effects that the modulator would have upon targets even in the absence of its own treatment; the effects of the modulation treatment are built on top of those unconditioned effects. If so, it is responding to the compound of the target and a control stimulus that provides the appropriate baseline against which to assess the effects of the modulator.

Unfortunately, it is not entirely clear what treatments one should adopt as the optimal control for modulation. In both of the present experiments, the control stimulus and the modulator were presented in the same temporal relation with a target and with reinforcement, but the control stimulus was arranged to be uninformative with regard to reinforcement of the target. However, this may have also resulted in differences other than the establishment of modulation. For instance, the control stimuli and modulators may have differed in the amount of excitatory strength they controlled. But it is worth noting that the two control treatments used here differed substantially in their own relations with the US. The fact that they produced quite similar effects on the targets may suggest that such relations are not of substantial importance. In any case, it seems clear that if there are substantial unconditioned effects of the modulator, then simply comparing responding to the target with and without the modulator could be seriously misleading; comparison with some control stimulus is essential. It is surprisingly frequent that studies of modulation continue to omit such a comparision.

\section{Independence of Path}

Finally, the present experiments add further to the list of results that suggest that one cannot represent the state of a stimulus simply by looking at its current response strength independently of its detailed conditioning history. Several results indicate that different ways of achiev- 
ing the same conditioning performance result from different learning representations. For instance, Brown-Su, Matzel, Gordon, and Miller (1986) have presented evidence that conditioned suppression achieved by a few trials with a strong US or by many trials with a weak US may be based on different underlying associations despite similar levels of performance.

The most frequently discussed case of this sort comes from one type of target stimuli studied here, extinguished stimuli. Many phenomena are taken as indicating that a trained and extinguished stimulus that generates responding similar to that of an untrained stimulus nevertheless does so on the basis of a different associative structure. Spontaneous recovery from extinction and disinhibition are the classic examples, but other examples include reinstatement from reinstitution of the US (Bouton \& Bolles, 1979b; Rescorla \& Heth, 1975) and renewal from change in context (e.g., Bouton \& Bolles, 1979a). Unfortunately, many studies examining these phenomena fail to match carefully the untreated current levels of performance of stimuli with various training histories (but see Bouton, 1984; Bouton \& King, 1986). Moreover, it is rare to compare two stimuli with different histories within the same animal, so as to control for the general treatment of the animal when examining the specific treatment of the stimulus. The contribution of the present experiments is that they do take this as their major goal and show quite different levels of responsivity from the same performance baseline. As such, they provide especially strong evidence for the importance of the history, as well as the present state, of the stimulus.

\section{REFERENCES}

Bombace, J. C., Brandon, S. E., \& Wagner, A. R. (1991). Modulation of a conditioned eyeblink response by a putative emotive stimulus conditioned with hindleg shock. Journal of Experimental Psychology: Animal Behavior Processes, 17, 323-333.

Bouton, M. E. (1984). Differential control by context in the inflation and reinstatement paradigms. Journal of Experimental Psychology: Animal Behavior Processes, 10, 56-74.

Bouton, M. E. (1991). Context and retrieval in extinction and in other examples of interference in simple associative learning. In L. W. Dachowski \& C. F. Flaherty (Eds.), Current topics in animal learning. Brain, emotion, and cognition (pp. 25-53). Hillsdale, NJ: Erlbaum.

Bouton, M. E., \& Bolles, R. C. (1979a). Contextual control of the extinction of conditioned fear. Learning \& Motivation, 10, 445-466.

Bouton, M. E., \& Bolles, R. C. (1979b). Role of conditioned contextual stimuli in reinstatement of extinguished fear. Journal of Experimental Psychology: Animal Behavior Processes, 5, 368-378.

Bouton, M. E., \& KING, D. A. (1986). Effect of context on performance to conditioned stimuli with mixed histories of reinforcement and nonreinforcement. Journal of Experimental Psychology: Animal Behavior Processes, 12, 4-15.

Bouton, M. E., \& SwartzenTRUber, D. (1989). Slow reacquisition following extinction: Context, encoding, and retrieval mechanisms. Journal of Experimental Psychology: Animal Behavior Processes, 15, 43-53.

Brown-Su, A. M., Matzel, L. D., Gordon, E. L., \& Miller, R. R.
(1986). Malleability of conditioned associations: Path dependence. Journal of Experimental Psychology: Animal Behavior Processes, 12, 420-427.

Davidson, T. L., \& JARRARD, L. E. (1989). Retention of concurrent conditional discriminations in rats with ibotenate lesions of hippocampus. Psychobiology, 17, 49-60.

Holland, P. C. (1983). Occasion setting in Pavlovian feature positive discriminations. In M. L. Commons, R. J. Herrnstein, \& A. R. Wagner (Eds.), Quantitative analyses of behavior: Discrimination processes (Vol. 4, pp. 183-206). New York: Ballinger.

Holland, P. C. (1985). The nature of conditioned inhibition in serial and simultaneous feature negative discriminations. In R. R. Miller $\&$ N. E. Spear (Eds.), Information processing in animals: Conditioned inhibition (pp. 267-297). Hillsdale, NJ: Erlbaum.

Holland, P. C. (1986). Transfer after serial feature positive discrimination training. Learning \& Motivation, 17, 243-268.

Holland, P. C. (1989a). Acquisition and transfer of conditional discrimination performance. Journal of Experimental Psychology: Animal Behavior Processes, 15, 154-165.

Holland, P. C. (1989b). Transfer of negative occasion setting and conditioned inhibition across conditioned and unconditioned stimuli. Journal of Experimental Psychology: Animal Behavior Processes, 15, 311-328.

Holland, P. C. (1991). Acquisition and transfer of occasion setting in operant feature positive and feature negative discriminations. Learning \& Motivation, 22, 366-387.

Holland, P. C., \& LAMARRE, J. (1984). Transfer of inhibition after serial and simultaneous feature negative discrimination training. Learning \& Motivation, 15, 219-243.

Holland, P. C., \& ReEve, C. E. (1991). Acquisition and transfer of control by an ambiguous cue. Animal Learning \& Behavior, 19, 113-124.

HoNeY, R. C., \& HaLl, G. (1989). Acquired equivalence and distinctiveness of cues. Journal of Experimental Psychology: Animal Behavior Processes, 15, 338-346.

KONORSKI, J. (1948). Conditioned reflexes and neuron organization. Cambridge: Cambridge University Press.

LamarRe, J., \& Holland, P. C. (1987). Transfer of inhibition after serial feature negative discrimination training. Learning \& Motivation, 18, 319-342.

Pearce, J. M. (1987). A model for stimulus generalization in Pavlovian conditioning. Psychological Review, 84, 61-73.

Rescorla, R. A. (1985). Conditioned inhibition and facilitation. In R. R. Miller \& N. E. Spear (Eds.), Information processing in animals: Conditioned inhibition (pp. 299-326). Hillsdale, NJ: Erlbaum.

RESCORLA, R. A. (1989). Simultaneous and sequential conditioned inhibition in autoshaping. Quarterly Journal of Experimental Psychology, 41B, 275-286.

RESCORLA, R. A. (1991). Separate reinforcement can enhance the effectiveness of modulators. Journal of Experimental Psychology: Animal Behavior Processes, 17, 259-269.

Rescorla, R. A. (1993). Interference among modulators. Animal Learning \& Behavior, 21, 179-186.

Rescorla, R. A., \& HETH, C. D. (1975). Reinstatement of fear to an extinguished stimulus. Journal of Experimental Psychology: Animal Behavior Processes, 1, 88-96.

ResCorla, R. A., \& WAGNER, A. R. (1972). A theory of Pavlovian conditioning: Variations in the effectiveness of reinforcement and nonreinforcement. In A. H. Black \& W. F. Prokasy (Eds.), Classical conditioning II: Current research and theory (pp. 64-99). New York: Appleton-Century-Crofts.

(Manuscript received June 23, 1993; revision accepted for publication February 2, 1994.) 